\title{
Normal Hyperbolicity and Continuity of Global Attractors for a Nonlocal Evolution Equations
}

\author{
Severino Horácio da Silva, ${ }^{1}$ Jocirei Dias Ferreira, ${ }^{2}$ and Flank David Morais Bezerra ${ }^{3}$ \\ ${ }^{1}$ Unidade Acadêmica de Matemática UAMAT/CCT/UFCG, Bairro Universitário, Rua Aprígio Veloso 882, \\ 58429-900 Campina Grande, PB, Brazil \\ ${ }^{2}$ Insituto de Ciencias Exatas e da Terra, Universidade Federal de Mato Grosso, Campus Universitário, Rodovia MT-100 Km 3,5, \\ 78.698-000 Barra do Garças, MT, Brazil \\ ${ }^{3}$ Departamento de Matemática UFPB/CCEN, Cidade Universitária, Campus I, 58051-900 João Pessoa, PB, Brazil
}

Correspondence should be addressed to Severino Horácio da Silva; horaciousp@gmail.com

Received 12 November 2013; Revised 4 March 2014; Accepted 4 March 2014; Published 24 April 2014

Academic Editor: Tuncay Candan

Copyright (c) 2014 Severino Horácio da Silva et al. This is an open access article distributed under the Creative Commons Attribution License, which permits unrestricted use, distribution, and reproduction in any medium, provided the original work is properly cited.

We show the normal hyperbolicity property for the equilibria of the evolution equation $\partial m(r, t) / \partial t=-m(r, t)+g(\beta J *$ $m(r, t)+\beta h), h, \beta \geq 0$, and using the normal hyperbolicity property we prove the continuity (upper semicontinuity and lower semicontinuity) of the global attractors of the flow generated by this equation, with respect to functional parameter $J$.

The first author dedicates this work to his daughter Luana Barros.

\section{Introduction}

We consider the nonlocal evolution equation

$$
\frac{\partial m(r, t)}{\partial t}=-m(r, t)+g(\beta J * m(r, t)+\beta h),
$$

where $m(r, t)$ is a real function on $\mathbb{R} \times \mathbb{R}_{+}, h$ and $\beta$ are nonnegative constants, and $J \in C^{1}(\mathbb{R})$ is a nonnegative even function supported in the interval $[-1,1]$ with integral equal to 1 . The $*$ denotes the convolution product, namely,

$$
(J * m)(x)=\int_{\mathbb{R}} J(x-y) m(y) d y .
$$

There are several works in the literature dedicated to the analysis of (1) or its particular case when $g \equiv \tanh$ (see [1-8]).

In the particular case when $g \equiv \tanh$, the existence of a compact global attractor for the flow of (1) was proved in [1] for bounded domain and $h=0$ and in [9] for unbounded domain.

If $g$ is globally Lipschitz, the Cauchy problem for (1) is well posed, for instance, in the space of continuous and bounded functions $C_{b}(\mathbb{R})$, with the sup norm since the function given by the right hand side of (1) is uniformly Lipschitz in this space (see $[10,11])$.

It is an easy consequence of the uniqueness theorem that the subspace $\mathbb{P}_{2 \tau}$ of the $2 \tau$ periodic functions is invariant for the flow of (1). We consider here (1) restricted to $\mathbb{P}_{2 \tau}$, with $\tau>$ 1. As shown in the previous work [7], this leads naturally to the consideration of the flow generated by $(1)$ in $L^{2}\left(S^{1}\right)$ where $S^{1}$ is the unit sphere and $*$ the convolution product in it. In what follows, we summarize the assumptions and results of [7]. For sake of clarity, it is convenient to start with a list of hypotheses satisfied by the function $g$.

(H1) The function $g: \mathbb{R} \rightarrow \mathbb{R}$ is globally Lipschitz; that is, there exists a positive constant $k_{1}$ such that

$|g(x)-g(y)| \leq k_{1}|x-y|, \quad$ for all $x, y \in \mathbb{R}$,

and there exist nonnegative constants $k_{2}$ and $k_{3}$, with $k_{2} \leq k_{1}$, such that

$$
|g(x)| \leq k_{2}|x|+k_{3}, \quad \text { for all } x \in \mathbb{R} .
$$


If $g$ is globally Lipschitz with constant $k_{1}$ it follows that (4) also holds with $k_{2}=k_{1}$ and $k_{3}=|g(0)|$. However, we are most interested in the case where $k_{2}<k_{1}$ because $k_{1} \beta<1$ can leave the attractor to the trivial case of only point.

(H2) The function $g \in C^{1}(\mathbb{R})$ and $g^{\prime}$ is Lipschitz with Lipschitz constant $k_{4}$. In particular, there exists a nonnegative constant $k_{5}$, such that

$$
\left|g^{\prime}(x)\right| \leq k_{4}|x|+k_{5}, \quad \text { for all } x \in \mathbb{R}
$$

(H3) The function $g$ has positive derivative.

(H4) There exists $a>0$ such that, for all $x \in \mathbb{R},|g(x)|<a$. In particular, when $a<\infty$ inequality (4) holds with $k_{2}=0$ and $k_{3}=a$.

(H5) The function $g^{-1}$ is continuous in $(-a, a)$ and the function

$$
f(m)=-\frac{1}{2} m^{2}-h m-\beta^{-1} i(m), \quad m \in[-a, a],
$$

where $i$ defined by

$$
i(m)=-\int_{0}^{m} g^{-1}(s) d s, \quad m \in[-a, a]
$$

has a global minimum $\bar{m}$ in $(-a, a)$.

Under hypothesis (H1) it was proved in [7] that the problem (1) is well posed in $L^{2}\left(S^{1}\right)$ and its flow is $C^{1}$ if we assume hypothesis (H2). Furthermore, assuming (H1) and (H2) the existence of a global compact attractor for the flow of (1) in the sense of [12] was also proved in [7]. A comparison result under the hypotheses $(\mathrm{H} 1)$ and $(\mathrm{H} 3)$ was also proved. Assuming (H1), (H2), (H3), and (H4), the authors in [7] showed an $L_{\infty}$ estimate for the attractors; finally, assuming (H5), they exhibited a continuous Lyapunov functional for the flow of (1) and proved under hypotheses (H1), (H2), (H3), (H4), and (H5) that its flow is gradient in the sense of [12].

The main purposes of this paper are showing normal hyperbolicity property of curves of equilibria and proving the continuity of global attractors for the flow of (1) with respect to the function $J$. To the extent of our knowledge, with the exception of [8], the proofs available in the literature concerning the continuity of global attractors assume that the equilibrium points of (1) are all hyperbolic and therefore isolated (see, e.g., [13-17]). However, this property cannot hold true in our case, due to the symmetries present in the equation. In fact, it is a consequence of these symmetries that the nonconstant equilibria arise in families and therefore it cannot be hyperbolic. To overcome this difficulty, in [8], the hypothesis of hyperbolicity of equilibria has been replaced by normal hyperbolicity of curves of equilibria.

The difference between our proof and the proof given in [8] is that in [8] the continuity with respect to scalar parameters $(h, \beta)$ is studied and here we study the continuity with respect to a functional parameter, namely, the function $J$. Moreover, in [8] it is assumed that the zero is a simple eigenvalue of the Frechét derivative of (8) which implies in normal hyperbolicity of curves of equilibria, and in this paper this property is also proven (see Propositions 12 and 14). To prove our results, we use some results given in [18] on the permanence of normally hyperbolic invariant manifolds and one result given in [19] concerning the continuity properties of the local unstable manifolds of the (nonnecessarily isolated) equilibria with respect to the parameter $J$, together with some results of [20] regarding the limiting behavior of the trajectories.

This paper is organized as follows. In Section 2, we show some preliminary results. Section 3 is devoted to the proof of the upper semicontinuity of the attractors. In Section 4, we show that families of equilibria are normally hyperbolic and we use this property to show the continuity of the families of equilibria with respect to the parameter. In Section 5, using the same techniques given in [8], we prove the lower semicontinuity of attractors. Finally, in Section 6, we illustrate our results with a concrete example.

\section{Background Results}

It is well known from [7] that under hypotheses (H1) and (H2) the map

$$
F(u, J)=-u+g(\beta(J * u)+\beta h)
$$

is continuously Frechet differentiable in $L^{2}\left(S^{1}\right)$, with $*$ being now the convolution product in $L^{2}\left(S^{1}\right)$; that is

$$
(J * v)(w)=\int_{S^{1}} J\left(w z^{-1}\right) v(z) d z
$$

Hence, the problem

$$
\frac{\partial u}{\partial t}=F(u, J)=-u+g(\beta(J * u)+\beta h)
$$

generates a $C^{1}$ flow in $L^{2}\left(S^{1}\right)$ which depends on the function $J$, which is given by the variation of constants formula

$$
u(w, t)=e^{-t} u(w, 0)+\int_{0}^{t} e^{-(t-s)}[g(\beta J * u(w, s)+\beta h)] d s
$$

From now on we denote this flow for problem $(\mathbf{P})_{J}$ by $T_{J}(t)$ or $T(J, t)$. It was proved in [7] that, in a certain range of the parameters, $T_{J}(t)$ admits a compact global attractor. Furthermore, assuming the hypotheses (H1)-(H5) we see that $T_{J}(t)$ has a gradient structure with Lyapunov functional $F$ : $L^{2}\left(S^{1}\right) \rightarrow \mathbb{R}$ given by

$$
\begin{aligned}
\mathbb{F}(u)= & \int_{S^{1}}[f(u(w))-f(\bar{m})] d w \\
& +\frac{1}{4} \iint_{S^{1}} J\left(w \cdot z^{-1}\right)[u(w)-u(z)]^{2} d w d z,
\end{aligned}
$$

where $f$ and $\bar{m}$ are given in the hypothesis (H5).

A natural question to examine is the dependence of the compact global attractor of $T_{J}(t)$ on the parameter $J$. 
We denote by $\mathscr{A}_{J}$ the global attractor of $(\mathbf{P})_{J}$ whose existence was proved in [7].

Let us recall that a family of subsets $\left\{\mathscr{A}_{J}\right\}$ is upper semicontinuous at $J_{0}$ if

$$
\operatorname{dist}\left(\mathscr{A}_{J}, \mathscr{A}_{J_{0}}\right) \longrightarrow 0, \quad \text { as } J \longrightarrow J_{0}
$$

where

$$
\operatorname{dist}\left(\mathscr{A}_{J}, \mathscr{A}_{J_{0}}\right)=\sup _{x \in \mathscr{A}_{J}} \operatorname{dist}\left(x, \mathscr{A}_{J_{0}}\right)=\sup _{x \in \mathscr{A}_{J}} \inf _{y \in \mathscr{A}_{J_{0}}}\|x-y\|_{L^{2}} .
$$

Analogously, $\left\{\mathscr{A}_{J}\right\}$ is lower semicontinuous at $J_{0}$ if

$$
\operatorname{dist}\left(\mathscr{A}_{J_{0}}, \mathscr{A}_{J}\right) \longrightarrow 0, \quad \text { as } J \longrightarrow J_{0}
$$

\section{Upper Semicontinuity of the Attractors}

In this section, we prove that the family of attractors $A_{J}$ is upper semicontinuous with respect to parameter $J$ at $J_{0}$, with $J, J_{0} \in \mathcal{J}$, where

$$
\begin{gathered}
\mathscr{J}=\left\{J \in C^{1}(\mathbb{R}),\right. \\
\text { even nonnegative, supported in }[-1,1], \\
\left.\|J\|_{L^{1}}=1, \text { and }\left\|J-J_{0}\right\|_{L^{1}} \leq R\right\} .
\end{gathered}
$$

Lemma 1. Assume that assumptions (H1) and (H2) hold and that $k_{2} \beta<1$. Then, the flow $T_{J}(t)$ is continuous with respect to $J$ in the $L^{1}$-norm at $J_{0}$, uniformly for $u$ in bounded sets and $t \in[0, b]$ with $b<\infty$.

Proof. As shown in [7] the solutions of $(\mathbf{P})_{J}$ satisfy the "variations of constants formula":

$$
T_{J}(t) u=e^{-t} u+\int_{0}^{t} e^{-(t-s)} g\left(\beta\left(J * T_{J}(s) u\right)+\beta h\right) d s .
$$

Let $J_{0} \in \mathcal{J}$. Given $\varepsilon>0$, we want to find $\delta>0$ such that $\left\|J-J_{0}\right\|_{L^{1}}<\delta$ implies

$$
\left\|T_{J}(t) u-T_{J_{0}}(t) u\right\|_{L^{2}}<\varepsilon
$$

for $t \in[0, b]$ and $u$ in $C$, where $C$ is a bounded set in $L^{2}\left(S^{1}\right)$. Since $g$ is globally Lipschitz, for any $t>0$ and $u \in C$, it follows that

$$
\begin{aligned}
& \left\|T_{J}(t) u-T_{J_{0}}(t) u\right\|_{L^{2}} \\
& \leq \int_{0}^{t} e^{-(t-s)} \| g\left(\beta J * T_{J}(s) u+\beta h\right) \\
& \quad-g\left(\beta J_{0} * T_{J_{0}}(s) u+\beta h\right) \|_{L^{2}} d s \\
& \leq \int_{0}^{t} e^{-(t-s)} k_{1}\left[\| \beta J *\left(T_{J}(s) u\right)\right. \\
& \left.\quad-\beta\left(J_{0} * T_{J_{0}}(s) u\right) \|_{L^{2}}\right] d s .
\end{aligned}
$$

Adding and subtracting the term $J_{0} * T_{J}(s) u$ inside the norm we get

$$
\begin{aligned}
&\left\|T_{J}(t) u-T_{J_{0}}(t) u\right\|_{L^{2}} \\
& \qquad \int_{0}^{t} e^{-(t-s)} k_{1} \beta\left[\left\|\left(J-J_{0}\right) * T_{J}(s) u\right\|_{L^{2}}\right. \\
&\left.\quad+\left\|J_{0} *\left(T_{J}(s) u-T_{J_{0}}(s) u\right)\right\|_{L^{2}}\right] d s .
\end{aligned}
$$

Using Young's inequality, we obtain

$$
\begin{aligned}
\| T_{J}(t) & u-T_{J_{0}}(t) u \|_{L^{2}} \\
\leq & k_{1} \beta\left\|J-J_{0}\right\|_{L^{1}} \int_{0}^{t} e^{-(t-s)}\left\|T_{J}(s) u\right\|_{L^{2}} d s \\
& +k_{1} \beta\left\|J_{0}\right\|_{L^{1}} \int_{0}^{t} e^{-(t-s)}\left\|T_{J}(s) u-T_{J_{0}}(s) u\right\|_{L^{2}} d s .
\end{aligned}
$$

From Theorem 3.3 of [7] it follows, for all nonnegative $J \in \mathscr{J}$, that if $k_{2} \beta<1$ and $(\mathrm{H} 1)$ and $(\mathrm{H} 2)$ hold then $\left\|T_{J}(t) u\right\|_{L^{2}}$ is bounded by a positive constant $K$ depending only on $C$. Thus, since $\left\|J_{0}\right\|_{L^{1}}=1$ we obtain

$$
\begin{aligned}
\| T_{J}(t) & u-T_{J_{0}}(t) u \|_{L^{2}} \\
\leq & K k_{1} \beta\left\|J-J_{0}\right\|_{L^{1}} \\
& \quad+k_{1} \beta \int_{0}^{t} e^{-(t-s)}\left\|T_{J}(s) u-T_{J_{0}}(s) u\right\|_{L^{2}} d s .
\end{aligned}
$$

Therefore, by Gronwall's lemma, it follows that

$$
\left\|T_{J}(t) u-T_{J_{0}}(t) u\right\|_{L^{2}} \leq C(J) e^{k_{1} \beta t}
$$

where $C(J)=K k_{1} \beta\left\|J-J_{0}\right\|_{L^{1}}$. This last assertion completes the proof.

Remark 2. Under hypotheses (H1) and (H2) and $k_{2} \beta<1$, from Theorem 3.3 of [7] it follows that, for all nonnegative $J \in \mathscr{J}$, there exists a global attractor $A_{J}$ in $L^{2}\left(S^{1}\right)$, which is contained in the ball with center at the origin of $L^{2}(S 1)$ and radius $2 \sqrt{2 \tau}\left(K_{2} \beta h+k_{3}\right) /\left(1-k_{2} \beta\right)$.

Now, using Remark 2 and proceeding as in [8], we obtain the following result.

Theorem 3. Assume that hypotheses (H1) and (H2) hold and that $k_{2} \beta<1$. Then the family of attractors $\mathscr{A}_{J}$ is upper semicontinuous with respect to $J \in \mathscr{J}$ at $J_{0}$.

\section{Normal Hyperbolicity and Lower Semicontinuity of the Attractors}

Due to the symmetries present in our model the nonconstant equilibria are nonisolated. In fact, as we will see shortly, the equivariance property of the map $F$ defined in (8) implies that the nonconstant equilibria appear in curves. Therefore, 
it cannot be hyperbolic preventing the use of tools like the Implicit Function Theorem to obtain their continuity with respect to the parameters. To overcome this difficulty, we need the concept of normal hyperbolicity (see [18]) and we also will need to assume the following additional hypotheses.

(H6) For each $J_{0} \in \mathcal{J}$, the set $E$ of the equilibria of $T_{J_{0}}(t)$ is such that $E=E_{1} \cup E_{2}$, where

(a) the equilibria in $E_{1}$ are constant hyperbolic equilibria;

(b) the equilibria in $E_{2}$ are nonconstant (consequently, nonhyperbolic).

(H7) The function $g \in C^{2}(\mathbb{R})$.

From hypotheses (H2) and (H7) it follows that $g^{\prime}$ is bounded; that is, there exists $b>0$ such that $\left|g^{\prime}(x)\right| \leq b$.

We start with some remarks on the spectrum of the linearization for $F$ around equilibria.

Remark 4. A straightforward calculation shows that if $u_{0}$ is nonconstant equilibria of $T_{J_{0}}(t)$ then zero is always an eigenvalue of the operator

$$
D F_{u}\left(u_{0}, J_{0}\right) v=-v+g^{\prime}\left(\beta J_{0} * u_{0}+\beta h\right) \beta\left(J_{0} * v\right)
$$

with eigenfunction $u_{0}^{\prime}$.

Remark 5. Let $u_{0} \in E_{2}$. It is easy to show that $D F_{u}\left(u_{0}, J_{0}\right)$ is a self-adjoint operator with respect to the inner product

$$
(u, v)=\int_{S^{1}} u(w) v(w) d v(w),
$$

where $d \nu(w)=d w / g^{\prime}\left(\beta\left(J_{0} * u_{0}\right)(w)+\beta h\right)$ is equivalent to the Lebesgue measure.

Remark 6. In [8] in the hypothesis (H6)-(b) it was also assumed as hypothesis that, for each $u_{0} \in E_{2}$, zero is simple eigenvalue of the operator $D F_{u}\left(u_{0}, J_{0}\right)$. However, in this paper, this property is proved (see Proposition 12).

In what follows we enunciated a result on the structure of the sets of nonconstant equilibria. The proof will be omitted since it is very similar to Lemma 3.3 in [8].

Lemma 7. Suppose that for some $J_{0} \in \mathcal{F},(H 1),(H 6)$, and (H7) hold. Given $u \in E_{2}$ and $\alpha \in S^{1}$, define $\gamma(\alpha ; u) \in L^{2}\left(S^{1}\right)$ by

$$
\gamma(\alpha ; u)(w)=u(\alpha w), \quad w \in S^{1} .
$$

Then $\Gamma=\gamma\left(S^{1} ; u\right)$ is a closed, simple $C^{2}$ curve of equilibria of $T_{J_{0}}(t)$ which is isolated in the set of equilibria; that is, no point of $\Gamma$ is an accumulation point of $E \backslash \Gamma$.

Corollary 8. Let $M$ be a closed connected curve of equilibria in $E_{2}$ and $u_{0} \in M$. Then $M=\Gamma$, where $\Gamma=\gamma\left(S^{1}, u_{0}\right)$.

Proof. Suppose that $\Gamma \not \subset M$. Then there exist equilibria in $M \backslash$ $\Gamma$ accumulating at $u_{0}$ contradicting Lemma 7. Therefore $\Gamma \subseteq$ $M$. Since $\Gamma$ is a simple closed curve, it follows that $M=\Gamma$.
The main results of this section will be presented in the next two subsections.

4.1. Normal Hyperbolicity of the Equilibria. Recall that if $T(t)$ : $X \rightarrow X$ is a semigroup a set $M \subset X$ is invariant under $T(t)$ if $T(t) M=M$, for any $t>0$.

Definition 9. Suppose that $T(t)$ is a $C^{1}$ semigroup in a Banach space $X$ and that $M \subset X$ is an invariant manifold for $T(t)$. We say that $M$ is normally hyperbolic under $T(t)$ if

(i) for each $m \in M$ there is a decomposition

$$
X=X_{m}^{c} \oplus X_{m}^{u} \oplus X_{m}^{s}
$$

by closed subspaces with $X_{m}^{c}$ being the tangent space to $M$ at $m$.

(ii) For each $m \in M$ and $t \geq 0$, if $m_{1}=T(t)(m)$

$$
\left.D T(t)(m)\right|_{X_{m}^{\alpha}}: X_{m}^{\alpha} \longrightarrow X_{m_{1}}^{\alpha}, \quad \alpha=c, u, s
$$

and $\left.D T(t)(m)\right|_{X_{m}^{u}}$ is an isomorphism from $X_{m}^{u}$ onto $X_{m_{1}}^{u}$.

(iii) There is $t_{0} \geq 0$ and $\mu<1$ such that for all $t \geq t_{0}$

$$
\begin{aligned}
\mu \inf & \left\{\left\|D T(t)(m) x^{u}\right\|: x^{u} \in X_{m}^{u},\left\|x^{u}\right\|=1\right\} \\
> & \max \left\{1,\left\|\left.D T(t)(m)\right|_{X_{m}^{c}}\right\|\right\}, \\
\mu \min & \left\{1, \inf \left\{\left\|D T(t)(m) x^{c}\right\|: x^{c} \in X_{m}^{c},\left\|x^{c}\right\|=1\right\}\right\} \\
> & \left\|\left.D T(t)(m)\right|_{X_{m}^{s}}\right\| .
\end{aligned}
$$

Condition (28) suggests that, near $m \in M, T(t)$ is expansive in the direction of $X_{m}^{u}$ and at rate greater than that on $M$, while (29) suggests that $T(t)$ is contractive in the direction of $X_{m}^{s}$ and at a rate greater than that on $M$.

The following result has been proved in [18].

Theorem 10 (normal hyperbolicity). Suppose that $T(t)$ is a $C^{1}$ semigroup on a Banach space $X$ and $M$ is a $C^{2}$ compact connected invariant manifold which is normally hyperbolic under $T(t)$ (i.e., (i) and (ii) of Definition 9 hold and there exists $0 \leq t_{0}<\infty$ such that (iii) holds for all $t \geq t_{0}$ ). Let $\widetilde{T}(t)$ be a $C^{1}$ semigroup on $X$ and $t_{1}>t_{0}$. Consider $N(\varepsilon)$, the $\varepsilon$ neighborhood of $M$, given by

$$
N(\varepsilon)=\left\{m+x^{u}+x^{s}, x^{u} \in X_{m}^{u}, x^{s} \in X_{m}^{s},\left\|x^{u}\right\|,\left\|x^{s}\right\|<\varepsilon\right\} .
$$

Then, there exists $\varepsilon^{*}>0$ such that, for each $\varepsilon<\varepsilon^{*}$, there exists $\sigma>0$ such that if

$$
\begin{aligned}
& \sup _{u \in N(\varepsilon)}\left\{\left\|\widetilde{T}\left(t_{1}\right) u-T\left(t_{1}\right) u\right\|\right. \\
&\left.+\left\|D \widetilde{T}\left(t_{1}\right)(u)-D T\left(t_{1}\right)(u)\right\|\right\}<\sigma, \\
& \sup _{u \in N(\varepsilon)}\|\widetilde{T}(t) u-T(t) u\|<\sigma, \quad \text { for } 0 \leq t \leq t_{1},
\end{aligned}
$$


there is a unique compact connected invariant manifold of class $C^{1}, \widetilde{M}$, in $N(\varepsilon)$. Furthermore, $\widetilde{M}$ is normally hyperbolic under $\widetilde{T}(t)$ and, for each $t \geq 0, \widetilde{T}(t)$ is a $C^{1}$-diffeomorphism from $\widetilde{M}$ to $\widetilde{M}$.

Remark 11. For $u \in L^{2}\left(S^{1}\right)$ we have

$$
\begin{aligned}
|(J * u)(w)| & \leq \int_{S^{1}}\left|J\left(w z^{-1}\right)\right||u(z)| d z \\
& \leq \int_{S^{1}}\|J\|_{\infty}|u(z)| d z \\
& \leq \sqrt{2 \tau}\|J\|_{\infty}\|u\|_{L^{2}},
\end{aligned}
$$

where we have used Hölder's inequality in the last estimate.

Motivated by [21] we prove below that, for each $u_{0} \in E_{2}$, zero is simple eigenvalue of $D F_{u}\left(u_{0}, J\right)$. But specifically we have the following result.

Proposition 12. Assume that $b \beta 2 \tau\|J\|_{\infty}<1$. Then, for each $u_{0} \in E_{2}$, zero is simple eigenvalue of $D F_{u}\left(u_{0}, J\right)$ with eigenfunction $u_{0}^{\prime}$.

Proof. From Remark 5, $D F_{u}\left(u_{0}, J\right)$ is self-adjoint operator. Then, to prove that zero is simple eigenvalue, it is enough to show that if $v \in \operatorname{ker}\left(D F_{u}\left(u_{0}, J\right)\right)$ then $v=\lambda_{0} u_{0}^{\prime}$, for some $\lambda_{0} \in \mathbb{R}$. For this, let $v \in L^{2}\left(S^{1}\right)$ be such that $D F_{u}\left(u_{0}, J\right) v=0$. Then

$$
v=g^{\prime}\left(\beta J * u_{0}+\beta h\right)(\beta J * v) .
$$

Suppose that, in $L^{2}\left(S^{1}\right), v \neq \lambda u_{0}^{\prime}$ for all $\lambda \in \mathbb{R}$; that is,

$$
\left\|v-\lambda u_{0}^{\prime}\right\|_{L^{2}}>0, \quad \forall \lambda \in \mathbb{R} \text {. }
$$

But, using Remark 11, for any $\lambda \in \mathbb{R}$ and almost every point of $S^{1}$, we have

$$
\begin{aligned}
\mid v(w)- & \lambda u_{0}^{\prime}(w) \mid \\
= & \mid g^{\prime}\left(\beta\left(J * u_{0}+\beta h\right)\right)(\beta J * v) \\
& \quad-\lambda g^{\prime}\left(\beta\left(J * u_{0}\right)+\beta h\right)\left(\beta J * u_{0}^{\prime}\right) \mid \\
= & \mid g^{\prime}\left(\beta\left(J * u_{0}+\beta h\right)\right)(\beta J * v) \\
& \quad-g^{\prime}\left(\beta\left(J * u_{0}\right)+\beta h\right)\left(\beta J *\left(\lambda u_{0}^{\prime}\right)\right) \mid \\
= & \left|g^{\prime}\left(\beta J * u_{0}+\beta h\right) \beta\left[J * v-J *\left(\lambda u_{0}^{\prime}\right)\right]\right| \\
= & \left|g^{\prime}\left(\beta J * u_{0}+\beta h\right)\right| \beta\left|J *\left[v-\lambda u_{0}^{\prime}\right]\right| \\
\leq & b \beta\|J\|_{\infty}\left\|v-\lambda u_{0}^{\prime}\right\|_{L^{2}} \sqrt{2 \tau} .
\end{aligned}
$$

Hence

$$
\left\|v-\lambda u_{0}^{\prime}\right\|_{L^{2}} \leq b \beta 2 \tau\|J\|_{\infty}\left\|v-\lambda u_{0}^{\prime}\right\|_{L^{2}} .
$$

Since $b \beta 2 \tau\|J\|_{\infty}<1$, and $\left\|v-\lambda u_{0}^{\prime}\right\|_{L^{2}}>0$, we obtain a contradiction. Therefore, there exists $\lambda_{0} \in \mathbb{R}$ such that $v=$ $\lambda u_{0}^{\prime}$.
Remark 13. Since

$$
v \longmapsto g^{\prime}\left(\beta J_{0} * u_{0}+\beta h\right) \beta\left(J_{0} * v\right)
$$

is a compact operator in $L^{2}\left(S^{1}\right)$, it follows from (H6) that

$$
\sigma\left(D F_{u}\left(u_{0}, J_{0}\right)\right) \backslash\{0\}
$$

contains only real eigenvalues of finite multiplicity with -1 as the unique possible accumulation point.

Proposition 14. Assume that the hypotheses (H1), (H2), and (H6) and that $b \beta\|J\|_{\infty} 2 \tau<1$ holds. Then, for each $J \in \mathcal{J}$, any curve of equilibria of $T_{J}(t)$ is a normally hyperbolic invariant manifold under $T_{J}(t)$.

Proof. Here we follow closely a proof given in Pereira and Silva [8]. Let $M$ be a curve of equilibria of $T_{J}(t)$ and $m \in M$. From Proposition 12 it follows that

$$
\operatorname{Ker}\left(D F_{u}(m, J)\right)=\operatorname{span}\left\{m^{\prime}\right\} .
$$

Let $Y=\mathscr{R}\left(D F_{u}(m, J)\right)$ be the range of $D F_{u}(m, J)$. Since $D F_{u}(m, J)$ is self-adjoint and Fredholm of index zero, it follows that

$$
\sigma\left(\left.D F_{u}\left(u_{0}, J\right)\right|_{Y}\right)=\sigma_{u} \cup \sigma_{s},
$$

where $\sigma_{u}$ and $\sigma_{s}$ correspond to the positive and negative eigenvalues, respectively.

From (H1) and (H2), it follows that $T_{J}(t)$ is a $C^{1}$ semigroup. Consider the linear autonomous equation

$$
\dot{v}=\left(\left.D F_{u}(m, J)\right|_{Y}\right) v .
$$

Then $D T_{J}(t) v_{0}$ is the solution of (41) with initial condition $v_{0}$; that is, $D T_{J}(t)(m) v_{0}=e^{\left(D F_{u}(m, J)\right) t} v_{0}$. In particular $\left.D T_{J}(t)(m)\right|_{Y} \equiv D\left(\left.T_{J}(t)\right|_{Y}\right)(m)=e^{\left(\left.D F_{u}(m, J)\right|_{Y}\right) t}$.

Let $P_{u}$ and $P_{s}$ be the spectral projections corresponding to $\sigma_{u}$ and $\sigma_{s}$. Thus, the subspaces $X_{m}^{u}=P_{u} Y$ and $X_{m}^{s}=P_{s} Y$ are invariant under $D T_{J}(t)$ and the following estimates hold (see $[11$, pages 73,81$]$ or $[22$, page 37$])$ :

$$
\begin{array}{cc}
\left\|\left.D T_{J}(t)\right|_{Y} v\right\| \leq N e^{-v t}\|v\|, & \text { for } v \in X_{m}^{s}, t \geq 0, \\
\left\|\left.D T_{J}(t)\right|_{Y} v\right\| \leq N e^{v t}\|v\|, & \text { for } v \in X_{m}^{u}, t \leq 0,
\end{array}
$$

for some positive constant $\nu$ and some constant $N>1$.

It is clear that $D T_{J}(t) \equiv 0$ when restricted to $X_{m}^{c}=$ $\operatorname{span}\left\{m^{\prime}\right\}$. Therefore, we have the decomposition

$$
L^{2}\left(S^{1}\right)=X_{m}^{c} \oplus X_{m}^{u} \oplus X_{m}^{s} .
$$

Since $\left.D F_{u}(m, J)\right|_{Y}$ is an isomorphism, then

$$
\left.D F_{u}(m, J)\right|_{X_{m}^{\alpha}}: X_{m}^{\alpha} \longrightarrow X_{m}^{\alpha}, \quad \alpha=u, s,
$$

is an isomorphism. Consequently, the linear flow

$$
\left.D T_{J}(t)(m)\right|_{X_{m}^{u}}: X_{m}^{u} \longrightarrow X_{m}^{u}
$$

is also an isomorphism.

Finally, the estimates (28) and (29) follow from estimate (42). 
Proposition 15. Suppose that the hypotheses (H1) and (H2) hold. Let $D T_{J}(t)(u)$ be the linear flow generated by the equation

$$
\frac{\partial v}{\partial t}=-v+g^{\prime}(\beta J * u+\beta h) \beta(J * v) .
$$

Then, for a fixed $J_{0} \in \mathcal{F}$, we have

$$
\begin{aligned}
& \left\|T_{J}(t) u-T_{J_{0}}(t) u\right\|_{L^{2}\left(S^{1}\right)} \\
& \quad+\left\|D T_{J}(t)(u)-D T_{J_{0}}(t)(u)\right\|_{\mathscr{L}\left(L^{2}\left(S^{1}\right), L^{2}\left(S^{1}\right)\right)} \longrightarrow 0,
\end{aligned}
$$

when $\left\|J-J_{0}\right\|_{L^{1}} \rightarrow 0$, uniformly for $u$ in bounded sets of $L^{2}\left(S^{1}\right)$ and $t \in[0, b], b<\infty$.

Proof. From Lemma 1 it follows that

$$
\left\|T_{J}(t) u-T_{J_{0}}(t) u\right\|_{L^{2}\left(S^{1}\right)} \longrightarrow 0, \quad \text { as }\left\|J-J_{0}\right\|_{L^{1}} \longrightarrow 0,
$$

for $u$ in bounded sets of $L^{2}\left(S^{1}\right)$ and $t \in[0, b]$.

By the variation of constants formula, we have

$$
\begin{aligned}
D T_{J}(t) & (u) v \\
& =e^{-t} v+\int_{0}^{t} e^{-(t-s)} g^{\prime}(\beta J * u+\beta h)(\beta J * v) d s .
\end{aligned}
$$

Thus

$$
\begin{aligned}
&\left\|D T_{J}(t)(u) v-D T_{J_{0}}(t)(u) v\right\|_{L^{2}} \\
& \leq \int_{0}^{t} e^{-(t-s)} \|\left[g^{\prime}(\beta J * u+\beta h) \beta(J * v)\right. \\
&\left.-g^{\prime}\left(\beta J_{0} * u+\beta h\right) \beta\left(J_{0} * v\right)\right] \|_{L^{2}} d s .
\end{aligned}
$$

Subtracting and adding the term $g^{\prime}(\beta J * u+\beta h) \beta\left(J_{0} * v\right)$, we have

$$
\begin{gathered}
\left\|D T_{J}(t)(u) v-D T_{J_{0}}(t)(u) v\right\|_{L^{2}} \\
\leq \int_{0}^{t} e^{-(t-s)}\left[\left\|g^{\prime}(\beta J * u+\beta h)\left[\beta\left(J-J_{0}\right) * v\right]\right\|_{L^{2}}\right. \\
+\|\left[g^{\prime}(\beta(J * u)+\beta h)\right. \\
\left.-g^{\prime}\left(\beta\left(J_{0} * u\right)+\beta h\right)\right] \\
\left.\times \beta\left(J_{0} * v\right) \|_{L^{2}}\right] d s .
\end{gathered}
$$

Now, using hypothesis (H2) and Remark 11, we obtain

$$
\begin{gathered}
\left\|g^{\prime}(\beta(J * u)+\beta h)\left[\beta\left(J-J_{0}\right) * v\right]\right\|_{L^{2}}^{2} \\
\leq \int_{S^{1}}\left[\beta k_{4}|(J * u)(w)|+\beta h+k_{5}\right]^{2} \\
\quad \times \beta^{2}\left|\left(J-J_{0}\right) * v(w)\right|^{2} d w \\
\leq \int_{S^{1}}\left[\beta k_{4} \sqrt{2 \tau}\|J\|_{\infty}\|u\|_{L^{2}}+k_{5}\right]^{2} \beta^{2} \\
\quad \times \int_{S^{1}}\left|\left(J-J_{0}\right) * v(w)\right|^{2} d w .
\end{gathered}
$$

Thus, by Young's inequality and from the fact that $u$ belongs to a bounded set (e.g., the ball in $L^{2}$ with radius $K$ ), it follows that

$$
\begin{aligned}
& \left\|g^{\prime}(\beta(J * u)+\beta h)\left[\beta\left(J-J_{0}\right) * v\right]\right\|_{L^{2}} \\
& \quad \leq\left[\beta k_{4} \sqrt{2 \tau}\|J\|_{\infty} K+k_{5}\right] \beta\left\|J-J_{0}\right\|_{L^{1}}\|v\|_{L^{2}} .
\end{aligned}
$$

From Remark 11 we obtain that

$$
\begin{gathered}
\left\|\left[g^{\prime}(\beta(J * u)+\beta h)-g^{\prime}\left(\beta\left(J_{0} * u\right)+\beta h\right)\right] \beta\left(J_{0} * v\right)\right\|_{L^{2}}^{2} \\
\leq \beta^{2}\left(\sqrt{2 \tau}\left\|J_{0}\right\|_{\infty}\|v\|_{L^{2}}\right)^{2} \\
\times \int_{S^{1}} \mid g^{\prime}(\beta(J * u)(w)+\beta h) \\
-\left.g^{\prime}\left(\beta\left(J_{0} * u(w)\right)+\beta h\right)\right|^{2} d w .
\end{gathered}
$$

Assuming (H2), Young's inequality, and the fact that $\|u\|_{L^{2}} \leq$ $K$ we get

$$
\begin{aligned}
\int_{S^{1}} \mid g^{\prime} & (\beta(J * u)(w)+\beta h)-\left.g^{\prime}\left(\beta\left(J_{0} * u(w)\right)+\beta h\right)\right|^{2} d w \\
\leq & \int_{S^{1}} k_{4}^{2}\left|\beta\left[\left(J-J_{0}\right) * u\right](w)\right|^{2} d w \\
\leq & k_{4}^{2} \beta^{2}\left[\left\|J-J_{0}\right\|_{L^{1}}\|u\|_{L^{2}}\right]^{2} \\
\leq & {\left[K k_{4} \beta\left\|J-J_{0}\right\|_{L^{1}}\right]^{2} . }
\end{aligned}
$$

Thus

$$
\begin{aligned}
& \left\|\left[g^{\prime}(\beta(J * u)+\beta h)-g^{\prime}\left(\beta\left(J_{0} * u\right)+\beta h\right)\right] \beta\left(\left(J_{0}\right) * v\right)\right\|_{L^{2}}^{2} \\
& \quad \leq \beta^{2}\left(\sqrt{2 \tau}\|J\|_{\infty}\right) K k_{4}\left\|J-J_{0}\right\|_{L^{1}}\|v\|_{L^{2}} .
\end{aligned}
$$

Hence, from (53) and (56) it follows that

$$
\begin{aligned}
\left\|D T_{J}(t)(u) v-D T_{J_{0}}(t)(u) v\right\|_{L^{2}} & \\
\leq & {\left[\left(K k_{4} \beta^{2} \sqrt{2 \tau}\|J\|_{\infty}+k_{5} \beta\right)\left\|J-J_{0}\right\|_{L^{1}}\right.} \\
& \left.+\beta\left(K k_{4} \beta \sqrt{2 \tau}\|J\|_{\infty}\right)\left\|J-J_{0}\right\|_{L^{1}}\right]\|v\|_{L^{2}} .
\end{aligned}
$$

Therefore

$$
\begin{aligned}
&\left\|D T_{J}(t)(u)-D T_{J_{0}}(t)(u)\right\|_{\mathscr{L}\left(L^{2}\left(S^{1}\right), L^{2}\left(S^{1}\right)\right)} \\
&=\sup _{\|v\|=1}\left\|D T_{J}(t)(u) v-D T_{J_{0}}(t)(u) v\right\|_{L^{2}\left(S^{1}\right)} \\
& \leq \sup _{\|v\|=1}\left[\left(K k_{4} \beta^{2} \sqrt{2 \tau}\|J\|_{\infty}+k_{5} \beta\right)\left\|J-J_{0}\right\|_{L^{1}}\right. \\
&\left.+\beta\left(K k_{4} \beta \sqrt{2 \tau}\|J\|_{\infty}\right)\left\|J-J_{0}\right\|_{L^{1}}\right]\|v\|_{L^{2}} .
\end{aligned}
$$


That is,

$$
\left\|D T_{J}(t)(u)-D T_{J_{0}}(t)(u)\right\|_{\mathscr{L}\left(L^{2}\left(S^{1}\right), L^{2}\left(S^{1}\right)\right)} \leq C(J),
$$

where

$$
\begin{aligned}
C(J)= & {\left[K k_{4} \beta^{2} \sqrt{2 \tau}\|J\|_{\infty}+k_{5} \beta\right]\left\|J-J_{0}\right\|_{L^{1}} } \\
& +\beta\left(K k_{4} \beta \sqrt{2 \tau}\|J\|_{\infty}\right)\left\|J-J_{0}\right\|_{L^{1}}
\end{aligned}
$$

tends to zero when $\left\|J-J_{0}\right\|_{L^{1}} \rightarrow 0$.

\subsection{Lower Semicontinuity of the Equilibria}

Theorem 16. Suppose that the hypotheses (H1), (H2), and (H5) with $a<\infty$ and (H6) and (H7) hold. Then, if $b \beta 2 \tau\|J\|_{\infty}<1$, the set $E_{J}$ of the equilibria of $T_{J}(t)$ is lower semicontinuous with respect to $J$ at $J_{0}$.

Proof. The continuity of the constant equilibria follows from the Implicit Function Theorem and the hypothesis of normal hyperbolicity.

Suppose now that $m$ is a nonconstant equilibrium of $(\mathbf{P})_{J}$ and let $\Gamma=\gamma(\alpha ; m)$ be the isolated curve of equilibria containing $m$ given in Lemma 7 . We wish to show that, for every $\varepsilon>0$, there exists $\delta>0$ so that if $J \in \mathcal{J}$ there exists $\Gamma_{J} \in E_{J}$ such that $\Gamma \subset \Gamma_{J}^{\varepsilon}$ where $\Gamma_{J}^{\varepsilon}$ is the $\varepsilon$-neighborhood of $\Gamma_{J}$.

From Lemma 7 and Propositions 14 and 15, the assumptions of the normal hyperbolicity theorem are satisfied. Thus, given $\varepsilon>0$, there is $\delta>0$ such that if $\left\|J-J_{0}\right\|_{L^{1}}<\delta$ there is a unique $C^{1}$ compact connected invariant manifold $\Gamma_{J}$ normally hyperbolic under $T_{J}(t)$, such that $\Gamma_{J}$ is $\varepsilon$-close and $C^{1}$-diffeomorphic to $\Gamma$.

Since $T_{J}(t)$ is gradient and $\Gamma_{J}$ is compact, there exists at least one equilibrium $m_{J} \in \Gamma_{J}$. In fact, the $\omega$ limit of any $u \in \Gamma_{J}$ is nonempty and belongs to $\Gamma_{J}$ by invariance. From Lemma 3.8.2 of [12], it must contain an equilibrium. Since $\Gamma_{J}$ is $\varepsilon$-close to $\Gamma$, there exists $m \in \Gamma$ such that $\left\|m-m_{J}\right\|_{L^{2}\left(S^{1}\right)}<\varepsilon$.

Let $\widetilde{\Gamma}_{J}$ be the curve of equilibria given by $\widetilde{\Gamma}_{J} \equiv$ $\left\{\gamma\left(\alpha ; m_{J}\right), \alpha \in S^{1}\right\}$ which is a normally hyperbolic invariant manifold under $T_{J}(t)$ by Proposition 14. Then, for each $\alpha \in$ $S^{1}$, we have

$$
\begin{aligned}
& \left\|\gamma\left(\alpha ; m_{J}\right)-\gamma(\alpha ; m)\right\|_{L^{2}}^{2} \\
& \quad=\int_{S^{1}}\left|\gamma\left(\alpha ; m_{J}\right)(w)-\gamma(\alpha ; m)(w)\right|^{2} d w \\
& \quad=\int_{S^{1}}\left|m_{J}(\alpha w)-m(\alpha w)\right|^{2} d w \\
& \quad=\left\|m_{J}-m\right\|_{L^{2}} .
\end{aligned}
$$

Thus

$$
\begin{aligned}
\left\|\gamma\left(\alpha ; m_{J}\right)-\gamma(\alpha ; m)\right\|_{L^{2}} & =\left\|m_{J}-m\right\|_{L^{2}} \\
& <\varepsilon .
\end{aligned}
$$

And $\Gamma$ is $\varepsilon$-close to $\widetilde{\Gamma}_{J}$. Since there are only a finite number of curves of equilibria the result follows immediately.
The example given below shows that the curves of equilibria of the equation

$$
\dot{x}=F(x),
$$

generated by the action of a group, may disappear even when the symmetry is preserved. In other words, we are unable to obtain a result by using the Implicit Function Theorem without additional hypotheses of normal hyperbolicity (see [23]).

Example 17 (an example with symmetry, see [8, 23]). Consider the planar system

$$
\begin{aligned}
& \dot{x}=x\left(1-x^{2}-y^{2}\right), \\
& \dot{y}=y\left(1-x^{2}-y^{2}\right) .
\end{aligned}
$$

Note that (64) has, besides the origin, the curve of equilibria given by

$$
x^{2}+y^{2}=1
$$

which is generated, in polar coordinate, by the rotation of a fixed equilibrium.

However, for any $\varepsilon \neq 0$, the perturbed system

$$
\begin{aligned}
& \dot{x}=-\varepsilon y+x\left(1-x^{2}-y^{2}\right), \\
& \dot{y}=\varepsilon x+y\left(1-x^{2}-y^{2}\right)
\end{aligned}
$$

has no nontrivial equilibrium, although the system is still invariant under the action of $S^{1}$.

\section{Lower Semicontinuity of the Attractors}

In this section, using the same techniques of [8] we present the proof of the lower semicontinuity of the attractors in the next two subsections below.

5.1. Existence and Continuity of the Local Unstable Manifolds. Let us return to $(\mathbf{P})_{J}$. Recall that the unstable set $W_{J}^{u}=W_{J}^{u}\left(u_{J}\right)$ of an equilibrium $u_{J}$ is the set of initial conditions $\varphi$ of $(\mathbf{P})_{J}$, such that $T_{J}(t) \varphi$ is defined for all $t \leq 0$ and $T_{J}(t) \varphi \rightarrow u_{J}$ as $t \rightarrow-\infty$. For a given neighborhood $V$ of $u_{J}$, the set $W_{J}^{u} \cap V$ is called a local unstable set of $u_{J}$.

In the following, using results of [19] we show that the local unstable sets are actually Lipschitz manifolds in a sufficiently small neighborhood and vary continuously with $J$. More precisely, we have the following.

Lemma 18. If $u_{0}$ is a fixed equilibrium of $(\mathbf{P})_{J}$ for $J=J_{0}$ then there is a $\delta>0$ such that if $\left\|J-J_{0}\right\|_{L^{1}}+\left\|u_{0}-u_{J}\right\|_{L^{2}}<\delta$ and

$$
U_{J}^{\delta}:=\left\{u \in W_{J}^{u}\left(u_{J}\right):\left\|u-u_{J}\right\|_{L^{2}}<\delta\right\}
$$

then $U_{J}^{\delta}$ is a Lipschitz manifold and

$$
\begin{array}{r}
\operatorname{dist}\left(U_{J}^{\delta}, U_{J_{0}}^{\delta}\right)+\operatorname{dist}\left(U_{J_{0}}^{\delta}, U_{J}^{\delta}\right) \longrightarrow 0 \\
\text { as }\left\|J-J_{0}\right\|_{L^{1}}+\left\|u_{0}-u_{J}\right\|_{L^{2}} \longrightarrow 0
\end{array}
$$

with dist defined as in (13). 
Proof. As already mentioned, assuming (H1) and (H2), the $\operatorname{map} F: L^{2}\left(S^{1}\right) \times \mathscr{J} \rightarrow L^{2}\left(S^{1}\right)$,

$$
F(u, J)=-u+g(\beta(J * u)+\beta h),
$$

defined by the right hand side of $(\mathbf{P})_{J}$ is continuously Frechet differentiable. Let $u_{J}$ be an equilibrium of $(\mathbf{P})_{J}$. Writing $u=$ $u_{J}+v$, it follows that $u$ is a solution of $(\mathbf{P})_{J}$ if and only if $v$ satisfies

$$
\frac{\partial v}{\partial t}=L(J) v+r\left(u_{J}, v, J\right),
$$

where $L(J) v=(\partial / \partial u) F\left(u_{J}, J\right)=-v+g^{\prime}\left(\beta\left(J * u_{J}\right)+\beta h\right) \beta(J * v)$ and $r\left(u_{J}, v, J\right)=F\left(u_{J}+v, J\right)-F\left(u_{J}, J\right)-L(J) v$. We rewrite (70) in the form

$$
\frac{\partial v}{\partial t}=L\left(J_{0}\right) v+f(v, J),
$$

where $f(v, J)=\left[L(J)-L\left(J_{0}\right)\right] v+r\left(u_{J}, v, J\right)$ is the "nonlinear part" of (71). Observe that now the "linear part" of (71) does not depend on the parameter $J$, as required by Theorems 2.5 and 3.3 from [19].

Note that

$$
\begin{aligned}
& \| g^{\prime}\left(\beta\left(J * u_{J}\right)+\beta h\right) \beta(J * v) \\
& -g^{\prime}\left(\beta\left(J_{0} * u_{\mathrm{J}_{0}}\right)+\beta h\right) \beta\left(J_{0} * v\right) \|_{L^{2}} \\
& \leq \|\left[g^{\prime}\left(\beta\left(J * u_{J}\right)+\beta h\right)-g^{\prime}\left(\beta\left(J_{0} * u_{J_{0}}\right)+\beta h\right)\right] \\
& \quad \times \beta(J * v) \|_{L^{2}} \\
& +\left\|g^{\prime}\left(\beta\left(J_{0} * u_{J_{0}}\right)+\beta h\right) \beta\left(J-J_{0}\right) * v\right\|_{L^{2}} .
\end{aligned}
$$

So, using (H2) and Young's inequality we obtain

$$
\begin{gathered}
\left\|\left[g^{\prime}\left(\beta\left(J * u_{J}\right)+\beta h\right)-g^{\prime}\left(\beta\left(J_{0} * u_{J_{0}}\right)+\beta h\right)\right] \beta(J * v)\right\|_{L^{2}} \\
=\left(\int_{S^{1}} \mid g^{\prime}\left(\beta\left(J * u_{J}\right)(w)+\beta h\right)\right. \\
-\left.g^{\prime}\left(\beta\left(J_{0} * u_{J_{0}}\right)(w)+\beta h\right)\right|^{2} \\
\left.\times \beta|(J * v)(w)|^{2} d w\right)^{1 / 2} \\
\leq \sqrt{2 \tau}\|J\|_{\infty} k_{4} \beta\|v\|_{L^{2}}\left\|J * u_{J}-J_{0} * u_{J_{0}}\right\|_{L^{2}},
\end{gathered}
$$

and consequently,

$$
\begin{aligned}
\left\|\left[g^{\prime}\left(\beta\left(J * u_{J}\right)+\beta h\right)-g^{\prime}\left(\beta\left(J_{0} * u_{J_{0}}\right)+\beta h\right)\right] \beta(J * v)\right\|_{L^{2}} \\
\leq \sqrt{2 \tau}\|J\|_{\infty} k_{4} \beta\|v\|_{L^{2}} \\
\quad \times\left[\left\|J *\left(u_{J}-u_{J_{0}}\right)\right\|_{L^{2}}+\left\|\left(J-J_{0}\right) * u_{J_{0}}\right\|_{L^{2}}\right] \\
\leq\left[\sqrt{2 \tau}\|J\|_{\infty} k_{4} \beta\|J\|_{L^{1}}\left\|u_{J}-u_{J_{0}}\right\|_{L^{2}}\right. \\
\left.\quad+\sqrt{2 \tau}\|J\|_{\infty} k_{4} \beta\left\|J-J_{0}\right\|_{L^{1}}\left\|u_{J_{0}}\right\|_{L^{2}}\right]\|v\|_{L^{2}} .
\end{aligned}
$$

On the other hand, since by hypothesis (H7) $g$ is $C^{2}$, the functions $g^{\prime}\left(\beta\left(J * u_{J}\right)+\beta(J * v)+\beta h\right)$ and $g^{\prime \prime}\left(\beta\left(J * u_{J}\right)+\right.$ $\beta(J * v)+\beta h)$ are bounded by a constant $M$; for any $J$ in a neighborhood of $J_{0}$ with $\|v\|_{L^{2}} \leq 1$, we have

$$
\begin{aligned}
& \left\|g^{\prime}\left(\beta\left(J_{0} * u_{J_{0}}\right)+\beta h\right) \beta\left(J-J_{0}\right) * v\right\|_{L^{2}} \\
& \quad \leq\left(\int_{S^{1}} M^{2} \beta^{2}\left|\left[\left(J-J_{0}\right) * v\right](w)\right|^{2} d w\right)^{1 / 2} \\
& \quad \leq M \beta\left\|J-J_{0}\right\|_{L^{1}}\|v\|_{L^{2}} .
\end{aligned}
$$

From (74) and (75) it follows that

$$
\begin{aligned}
& \|\left[g^{\prime}\left(\beta\left(J * u_{J}\right)+\beta h\right) \beta(J * v)\right. \\
& \left.-g^{\prime}\left(\beta\left(J_{0} * u_{J_{0}}\right)+\beta h\right)\right] \beta\left(J_{0} * v\right) \|_{L^{2}} \\
& =\left[\sqrt{2 \tau}\|J\|_{\infty} k_{4} \beta\|J\|_{L^{1}}\left\|u_{J}-u_{J_{0}}\right\|_{L^{2}}\right. \\
& \quad+\sqrt{2 \tau}\|J\|_{\infty} k_{4} \beta\left\|J-J_{0}\right\|_{L^{1}}\left\|u_{J_{0}}\right\|_{L^{2}} \\
& \left.\quad+M \beta\left\|J-J_{0}\right\|_{L^{1}}\right]\|v\|_{L^{2}} .
\end{aligned}
$$

Therefore,

$$
\begin{aligned}
& \left\|\left[L(J)-L\left(J_{0}\right)\right] v\right\|_{L^{2}} \\
& =\|\left[g^{\prime}\left(\beta\left(J * u_{J}\right)+\beta h\right) \beta(J * v)-g^{\prime}\left(\beta\left(J_{0} * u_{J_{0}}\right)+\beta h\right)\right] \\
& \quad \times \beta\left(J_{0} * v\right) \|_{L^{2}} \\
& \leq C_{1}(J)\|v\|_{L^{2}},
\end{aligned}
$$

where

$C_{1}(J)$

$$
\begin{aligned}
=\left[\sqrt{2 \tau}\|J\|_{\infty} k_{4} \beta\|J\|_{L^{1}}\left\|u_{J}-u_{J_{0}}\right\|_{L^{2}}\right. & \\
\left.+\sqrt{2 \tau}\|J\|_{\infty} k_{4} \beta\left\|J-J_{0}\right\|_{L^{1}}\left\|u_{J_{0}}\right\|_{L^{2}}+M \beta\left\|J-J_{0}\right\|_{L^{1}}\right] & \longrightarrow 0, \\
\text { as }\left\|J-J_{0}\right\|_{L^{1}} & \longrightarrow 0 .
\end{aligned}
$$

Now, note that

$$
\begin{aligned}
F\left(u_{J}(w)+v(w), J\right)-F\left(u_{J_{0}}(w)+v(w), J_{0}\right) \\
=\left[u_{J_{0}}-u_{J}\right] \\
+\left[g\left(\beta\left(J * u_{J}\right)(w)+\beta(J * v)(w)+\beta h\right)\right. \\
\left.\quad-g\left(\beta\left(J_{0} * u_{J_{0}}\right)(w)+\beta\left(J_{0} * v\right)(w)+\beta h\right)\right] \\
=\left[g\left(\beta\left(J_{0} * u_{J_{0}}\right)(w)+\beta h\right)\right. \\
\left.-g\left(\beta\left(J_{0} * u_{J_{0}}\right)(w)+\beta\left(J_{0} * v\right)(w)+\beta h\right)\right] \\
+\left[g\left(\beta\left(J * u_{J}\right)(w)+\beta(J * v)(w)+\beta h\right)\right. \\
\left.-g\left(\beta\left(J * u_{J}\right)(w)+\beta h\right)\right] .
\end{aligned}
$$


Thus

$$
\begin{aligned}
F\left(u_{J}\right. & (w)+v(w), J)-F\left(u_{J_{0}}(w)+v(w), J_{0}\right) \\
= & g^{\prime}\left(\beta\left(J * u_{J}\right)(w)+\beta(J * \bar{v})(w)+\beta h\right) \beta(J * v)(w) \\
& -g^{\prime}\left(\beta\left(J_{0} * u_{J_{0}}\right)(w)+\beta(J * \bar{v})(w)+\beta h\right) \\
& \times \beta\left(J_{0} * v\right)(w),
\end{aligned}
$$

for some $\bar{v}$ in the segment defined by $J * u_{J}$ and $J *\left(u_{J}+v\right)$ and for some $\overline{\bar{v}}$ in the segment defined by $J_{0} * u_{J_{0}}$ and $J_{0} *\left(u_{J_{0}}+v\right)$. Then, using (H2) and the fact that $g^{\prime}\left(\beta\left(J * u_{J}\right)+\beta(J * v)+\beta h\right)$ is bounded by a constant $M$, for any $J$ in a neighborhood of $J_{0}$ with $\|v\|_{L^{2}} \leq 1$, we have

$$
\begin{aligned}
F\left(u_{J}\right. & (w)+v(w), J)-F\left(u_{J_{0}}(w)+v(w), J_{0}\right) \\
= & g^{\prime}\left(\beta\left(J * u_{J}\right)(w)+\beta(J * \bar{v})(w)+\beta h\right) \\
& \times \beta(J * v)(w) \\
& -g^{\prime}\left(\beta\left(J_{0} * u_{J_{0}}\right)(w)+\beta\left(J_{0} * \bar{v}\right)(w)+\beta h\right) \\
& \times \beta(J * v)(w) \\
& +g^{\prime}\left(\beta\left(J_{0} * u_{J_{0}}\right)(w)+\beta\left(J_{0} * \bar{v}\right)(w)+\beta h\right) \\
& \times \beta(J * v)(w) \\
& -g^{\prime}\left(\beta\left(J_{0} * u_{J_{0}}\right)(w)+\beta(J * \bar{v})(w)+\beta h\right) \\
& \times \beta\left(J_{0} * v\right)(w) .
\end{aligned}
$$

With this

$$
\begin{aligned}
\left\|F\left(u_{J}+v, J\right)-F\left(u_{J_{0}}+v, J_{0}\right)\right\|_{L^{2}} \\
\leq M \beta\left\|\left[\left(J * u_{J}\right)-\left(J_{0} * u_{J_{0}}\right)+J *(\overline{\bar{v}}-\bar{v})\right] \beta(J * v)\right\| \\
\quad+M \beta\left\|J_{0} * \bar{v}-J * \overline{\bar{v}}\right\|_{L^{2}} \\
\leq M \beta\left\|\left[\left(J * u_{J}\right)-\left(J_{0} * u_{J_{0}}\right)+J *(\overline{\bar{v}}-\bar{v})\right] \beta(J * v)\right\| \\
\quad+M \beta\left\|\left(J-J_{0}\right) * v\right\|_{L^{2}}+M \beta\|J *(\bar{v}-\overline{\bar{v}})\|_{L^{2}} .
\end{aligned}
$$

Once the following estimates hold

$$
\begin{aligned}
& \left\|\left(J * u_{J}-J_{0} * u_{J_{0}}\right)(J * v)\right\|_{L^{2}} \\
& \leq \sqrt{2 \tau}\|J\|_{\infty} \\
& \quad \times\left(\|J\|_{L^{1}}\left\|u_{J}-u_{J_{0}}\right\|_{L^{2}}+\left\|u_{J_{0}}\right\|_{L^{2}}\left\|J-J_{0}\right\|_{L^{1}}\right)\|v\|_{L^{2}}, \\
& \|J *(\overline{\bar{v}}-\bar{v})(J * v)\|_{L^{2}} \\
& \quad \leq \sqrt{2 \tau}\|J\|_{\infty}\left(\|J\|_{L^{1}}\|\overline{\bar{v}}-\bar{v}\|_{L^{2}}\right)\|v\|_{L^{2}}, \\
& \left\|\left[\left(J-J_{0}\right) * v\right](w)\right\|_{L^{2}} \leq\left\|J-J_{0}\right\|_{L^{1}}\|v\|_{L^{2}},
\end{aligned}
$$

it follows that

$$
\begin{aligned}
& \left\|F\left(u_{J}+v, J\right)-F\left(u_{J_{0}}+v, J_{0}\right)\right\|_{L^{2}} \\
& \leq M \beta^{2} \sqrt{2 \tau}\|J\|_{\infty}\|J\|_{L^{1}} \\
& \quad \times\left(k_{4}\left\|u_{J}-u_{J_{0}}\right\|_{L^{2}}+\|\overline{\bar{v}}-\bar{v}\|_{L^{2}}\right)\|v\|_{L^{2}} \\
& \quad+\left(M \beta^{2} \sqrt{2 \tau}\|J\|_{\infty}\left\|u_{J_{0}}\right\|_{L^{2}}\left\|J-J_{0}\right\|_{L^{1}}+M \beta\left\|J-J_{0}\right\|_{L^{1}}\right) \\
& \quad \times\|v\|_{L^{2}} .
\end{aligned}
$$

Therefore, as $\|\bar{v}-\overline{\bar{v}}\|_{L^{2}} \rightarrow 0$ provided that $\left\|J-J_{0}\right\|_{L^{2}} \rightarrow 0$, it follows that

$$
\left\|F\left(u_{J}+v, J\right)-F\left(u_{J_{0}}+v, J_{0}\right)\right\|_{L^{2}} \leq C_{2}(J)\|v\|_{L^{2}},
$$

with $C_{2}(J) \rightarrow 0$ when $\left\|J-J_{0}\right\|_{L^{2}} \rightarrow 0$.

Since $r\left(u_{J}, v, J\right)=F\left(u_{J}+v, J\right)-L(J) v$, we obtain from (77) and (85) that

$$
\left\|r\left(u_{J}, v, J\right)-r\left(u_{J_{0}}, v, J\right)\right\| \leq C_{3}(J)\|v\|_{L^{2}} .
$$

From (77) and (86), it follows that

$$
\left\|f(v, J)-f\left(v, J_{0}\right)\right\| \leq C_{4}(J)\|v\|_{L^{2}},
$$

where $C_{4}(J) \rightarrow 0$ as $\left\|J-J_{0}\right\|_{L^{2}} \rightarrow 0$.

In a similar way, we obtain that

$$
\begin{aligned}
& \left|r\left(u_{J}(z), v(z), J\right)-r\left(u_{J}(z), w(z), J\right)\right| \\
& \leq\left\|g^{\prime \prime}\left(\beta J * u_{J}(z)+\beta J * \overline{\bar{v}}(z)+\beta h\right) \beta J * \bar{v}(z)\right\|_{\infty} \\
& \quad \times \beta^{2} \sqrt{4 \tau^{2}}\|J\|_{\infty}^{2}\|\bar{v}\|_{L^{2}}\|v-w\|_{L^{2}},
\end{aligned}
$$

for any $v, w$ with $\|v\|_{L^{2}\left(S^{1}\right)}$ and $\|w\|_{L^{2}\left(S^{1}\right)}$ smaller than 1 , with $\bar{v}$ in the segment defined by $\beta J * v+\beta h$ and $\beta J * w+\beta h$ and $\overline{\bar{v}}$ in the segment defined by 0 and $\beta(J * \bar{v})+\beta h$. As $\|v\|_{L^{2}},\|w\|_{L^{2}} \rightarrow$ 0 , it follows that

$$
\begin{aligned}
& \left\|r\left(u_{J}(z), v(z), J\right)-r\left(u_{J}(z), w(z), J\right)\right\|_{L^{2}} \\
& \leq v_{1}(\rho)\|v-w\|_{L^{2}},
\end{aligned}
$$

with $v(\rho) \rightarrow 0$ when $\rho \rightarrow 0$ and $\|v\|_{L^{2}},\|w\|_{L^{2}}<\rho$. Furthermore

$$
\begin{gathered}
\left\|\left[L(J)-L\left(J_{0}\right)\right] v-\left[L(J)-L\left(J_{0}\right)\right] w\right\|_{L^{2}} \\
\leq C_{1}(J)\|(v-w)\|_{L^{2}} .
\end{gathered}
$$

Thus

$$
\|f(v, J)-f(w, J)\|_{L^{2}} \leq\left(\nu(\rho)+C_{1}(J)\right)\|v-w\|_{L^{2}},
$$

with $\nu(\rho) \rightarrow 0$ when $\rho \rightarrow 0,\|v\|_{L^{2}}$ and $\|w\|_{L^{2}}$ are less than or equal to $\rho$, and $C_{1}(J) \rightarrow 0$ when $J \rightarrow J_{0}$.

Therefore, the conditions of Theorems 2.5 and 3.3 from [19] are satisfied and we obtain the existence of locally invariant sets for (71) near the origin, given as graphics of 
Lipschitz functions which depend continuously on the parameter $J$ near $J_{0}$. Using uniqueness of solutions, we can easily prove that these sets coincide with the local unstable manifolds of (71).

Observing now that the translation

$$
u \longmapsto\left(u-u_{J}\right)
$$

sends an equilibrium $u_{J}$ of $(\mathbf{P})_{J}$ into the origin (which is an equilibrium of (71)), the results follow immediately.

Using the compactness of the set of equilibria, one can obtain a "uniform version" of Lemma 18 that will be needed later.

Lemma 19. Let $J=J_{0}$ be fixed. Then, there exists a $\delta>$ 0 such that, for any equilibrium $u_{0}$ of $(P)_{J_{0}}$, if $\left\|J-J_{0}\right\|_{L^{1}}+$ $\left\|u_{0}-u_{J}\right\|_{L^{2}}<\delta$ and

$$
U_{J}^{\delta}:=\left\{u \in U_{J}\left(u_{J}\right):\left\|u-u_{J}\right\|_{L^{2}\left(S^{1}\right)}<\delta\right\}
$$

then $U_{J}^{\delta}$ is a Lipschitz manifold and

$$
\begin{array}{r}
\sup _{u_{0} \in E_{J_{0}}} \operatorname{dist}\left(U_{J}^{\delta}, U_{J_{0}}^{\delta}\right)+\operatorname{dist}\left(U_{J_{0}}^{\delta}, U_{J}^{\delta}\right) \longrightarrow 0 \\
\text { as }\left\|J-J_{0}\right\|_{L^{1}}+\left\|u_{0}-u_{J}\right\|_{L^{2}} \longrightarrow 0,
\end{array}
$$

with dist defined as in (13).

Proof. From Lemma 18 we know that, for any $u_{0} \in E_{J_{0}}$, there exists a $\delta=\delta\left(u_{0}\right)$ such that $U_{J}^{\delta}$ is a Lipschitz manifold if $\left\|J-J_{0}\right\|_{L^{1}}+\left\|u_{0}-u_{J}\right\|_{L^{2}}<2 \delta$. In particular, $U_{J}^{\delta}$ is a Lipschitz manifold if $\left\|J-J_{0}\right\|_{L^{1}}+\left\|\widetilde{u}_{0}-u_{J}\right\|_{L^{2}}<\delta$ for any $\widetilde{u}_{0} \in E_{J_{0}}$ with $\left\|\tilde{u}_{0}-u_{0}\right\|_{L^{2}}<\delta$. Taking a finite subcovering of the covering of $E_{J_{0}}$ by balls $B\left(u_{0}, \delta\left(u_{0}\right)\right)$, with $u_{0}$ varying in $E_{J_{0}}$, the first part of the result follows with $\delta$ chosen as the minimum of those $\delta\left(u_{0}\right)$.

Now, if $\varepsilon>0$ and $u_{0} \in E_{J_{0}}$ there exists, by Lemma 18, $\delta=\delta\left(u_{0}\right)$ such that if $\left\|J-J_{0}\right\|_{L^{1}}+\left\|u_{0}-u_{J}\right\|_{L^{2}}<2 \delta$ then

$$
\operatorname{dist}\left(U_{J}^{\delta}, U_{J_{0}}^{\delta}\right)+\operatorname{dist}\left(U_{J 0}^{\delta}, U_{J}^{\delta}\right)<\frac{\varepsilon}{2} .
$$

If $\tilde{u}_{0} \in E_{J_{0}}$ is such that $\left\|\tilde{u}_{0}-u_{0}\right\|_{L^{2}}<\delta$ and $\left\|J-J_{0}\right\|_{L^{1}}+$ $\left\|\tilde{u}_{0}-u_{J}\right\|_{L^{2}}<\delta$ then, since $\left\|J-J_{0}\right\|_{L^{1}}+\left\|u_{0}-u_{J}\right\|_{L^{2}}<2 \delta$,

$$
\begin{aligned}
& \operatorname{dist}\left(U_{J}^{\delta}\left(u_{J}\right), U_{J_{0}}^{\delta}\left(\tilde{u}_{0}\right)\right)+\operatorname{dist}\left(U_{J_{0}}^{\delta}\left(\tilde{u}_{0}\right), U_{J}^{\delta}\left(u_{J}\right)\right) \\
&< \operatorname{dist}\left(U_{J}^{\delta}\left(u_{J}\right), U_{J_{0}}^{\delta}\left(u_{0}\right)\right)+\operatorname{dist}\left(U_{J_{0}}^{\delta}\left(u_{0}\right), U_{J}^{\delta}\left(u_{J}\right)\right) \\
& \quad+\operatorname{dist}\left(U_{J_{0}}^{\delta}\left(\widetilde{u}_{0}\right), U_{J_{0}}^{\delta}\left(u_{0}\right)\right) \\
& \quad+\operatorname{dist}\left(U_{J_{0}}^{\delta}\left(u_{0}\right), U_{J_{0}}^{\delta}\left(\tilde{u}_{0}\right)\right)<\varepsilon .
\end{aligned}
$$

By the same procedure given above, taking a finite subcovering of the covering of $E_{J_{0}}$ by balls $B\left(u_{0}, \delta\left(u_{0}\right)\right)$ and $\delta$ the minimum of those $\delta\left(u_{0}\right)$, we conclude that

$$
\begin{aligned}
& \quad \operatorname{dist}\left(U_{J}^{\delta}\left(u_{J}\right), U_{J_{0}}^{\delta}\left(\widetilde{u}_{0}\right)\right)+\operatorname{dist}\left(U_{J_{0}}^{\delta}\left(\tilde{u}_{0}\right), U_{J}^{\delta}\left(u_{J}\right)\right)<\varepsilon \\
& \text { if }\left\|J-J_{0}\right\|_{L^{1}}+\left\|\widetilde{u}_{0}-u_{J}\right\|_{L^{2}}<\delta \text { for any } \tilde{u}_{0} \in E_{J_{0}} .
\end{aligned}
$$

5.2. Characterization and Proof of Lower Semicontinuity of the Attractors. As a consequence of its gradient structure (see [7]), the attractor of the flow generated by $(\mathbf{P})_{J}$ is given by unstable set of the set of equilibria (see [12]). Using results of [20], we obtain below a more precise characterization of the attractors.

Consider an equation of the form

$$
\dot{x}+B x=g(x),
$$

where $B$ is a bounded linear operator on a Banach space $X$ and $g: X \rightarrow X$ is a $C^{2}$ function. We may write (98) in the form

$$
\dot{x}+A x=f(x),
$$

where $A=B-g^{\prime}\left(x_{0}\right)$ and $f(x)=g\left(x_{0}\right)+r(x)$, with $r$ differentiable and $r(0)=0$.

The following result has been proved in [20].

Theorem 20. Suppose that the spectrum $\sigma(A)$ contains 0 as a simple eigenvalue, while the remainder of the spectrum has real part outside some neighborhood of zero. Let $\gamma$ be a $C^{2}$ curve of equilibria of the flow generated by (99). Then there exists a neighborhood $U$ of $\gamma$ such that, for any $x_{0} \in U$ whose positive orbit is precompact and whose $\omega$-limit set $\omega\left(x_{0}\right)$ belongs to $\gamma$, there exists a unique point $y\left(x_{0}\right) \in \gamma$ with $\omega\left(x_{0}\right)=y\left(x_{0}\right)$. Similarly, for any $x_{0} \in U$ with bounded negative orbit and $\alpha$ limit set $\alpha\left(x_{0}\right)$ in $\gamma$, there exists a unique point $y\left(x_{0}\right) \in \gamma$ such that $\alpha\left(x_{0}\right)=y\left(x_{0}\right)$.

Proposition 21. Assume that the hypotheses (H1), (H2), and (H5) with $a<\infty$ and (H6) and (H7) hold. Let $E_{J}$ be the set of equilibria for $T_{J}(t)$. For $u \in E_{J}$, let $W_{J}^{u}(u)$ be the unstable set of $u$. Then the attractor of the flow $T_{J}(t)$ is given by

$$
\mathscr{A}_{J}=\bigcup_{u \in E_{J}} W_{J}^{u}(u) \text {. }
$$

Proof. From Theorem 5.5 of [7] we have

$$
\mathscr{A}_{J}=\bigcup_{u \in E_{J}} W_{J}^{u}\left(E_{J}\right)
$$

There exist only a finite number $\left\{u_{1}, \ldots, u_{k}\right\}$ of constant equilibria since they are all hyperbolic. For each nonconstant equilibrium $u \in E_{J}$, there is a curve $M_{u} \subset E_{J} \subset \mathscr{A}_{J}$. From Lemma 7 these curves $M_{u}$ are all isolated and, since $\mathscr{A}_{J}$ is compact, it follows that there exist only a finite number of them, namely, $M_{1}, \ldots, M_{n}$. Thus

$$
\mathscr{A}_{J}=\left(\bigcup_{i=1}^{n} W_{J}^{u}\left(M_{i}\right)\right) \bigcup\left(\bigcup_{j=1}^{k} W_{J}^{u}\left(u_{j}\right)\right) \text {. }
$$

By Theorem 20, it follows that

$$
W_{J}^{u}\left(M_{i}\right)=\bigcup_{v \in M_{i}} W_{J}^{u}(v), \quad i=1, \ldots, n .
$$

Therefore

$$
\mathscr{A}_{J}=\bigcup_{v \in E_{J}} W_{J}^{u}(v) .
$$


5.2.1. Proof of the Lower Semicontinuity. We now turn back to the proof of our main result, starting with some auxiliary results.

Lemma 22. Assume the same hypotheses of Proposition 21. Thus, given $\varepsilon>0$ there exists $T>0$ such that, for all $u \in$ $\mathscr{A}_{J_{0}} \backslash E_{J_{0}}^{\varepsilon}$,

$$
T_{J_{0}}(-t) u \in E_{J_{0}}^{\varepsilon},
$$

for some $t \in[0, T]$, where $E_{J_{0}}^{\varepsilon}$ is the $\varepsilon$-neighborhood of $E_{J_{0}}$. Furthermore, when $\varepsilon$ is sufficiently small,

$$
T_{J_{0}}(-t) u \in U_{J_{0}}\left(u_{0}\right)
$$

for some $u_{0} \in E_{J_{0}}$, where $U_{J_{0}}\left(u_{0}\right)$ is the local unstable manifold of $u_{0} \in E_{J_{0}}$.

Proof. Let $\varepsilon>0$ be given and $u \in \mathscr{A}_{J_{0}} \backslash E_{J_{0}}^{\varepsilon}$. From Proposition 21, it follows that

$$
u \in W_{J_{0}}^{u}(\bar{u}) \backslash E_{J_{0}}^{\varepsilon}
$$

for some $\bar{u} \in E_{J_{0}}$. Thus, there exists $t_{u}=t_{u}(\varepsilon)<\infty$ such that $T_{J_{0}}\left(-t_{u}\right) u \in E_{J_{0}}^{\varepsilon}$. By the continuity of the operator $T_{J_{0}}\left(-t_{u}\right)$, there exists $\eta_{u}>0$ such that $T_{J_{0}}\left(-t_{\mathrm{u}}\right) B\left(u, \eta_{u}\right) \subset E_{J_{0}}^{\varepsilon}$, where $B\left(u, \eta_{u}\right)$ is the ball of center $u$ and radius $\eta_{u}$. By compactness, there are $u_{1}, \ldots, u_{n} \in \mathscr{A}_{J_{0}} \backslash E_{J_{0}}^{\mathcal{E}}$ such that

$$
\mathscr{A}_{J_{0}} \backslash E_{J_{0}}^{\mathcal{\varepsilon}} \subset \bigcup_{j=1}^{n} B\left(u_{j}, \eta_{u_{j}}\right)
$$

with $T_{J_{0}}\left(-t_{u_{j}}\right) B\left(u_{j}, \eta_{u_{j}}\right) \subset E_{J_{0}}^{\varepsilon}$, for $j=1, \ldots, n$. Let $T=$ $\max \left\{t_{u_{1}}, \ldots, t_{u_{n}}\right\}$. Then, for any $u \in \mathscr{A}_{0} \backslash E_{J_{0}}^{\varepsilon}, T_{J_{0}}(-t) u \in E_{J_{0}}^{\varepsilon}$ for some $t \in[0, T]$. Since $u \in W_{J_{0}}^{u}(\bar{u}) \backslash E_{J_{0}}^{\mathcal{E}}$ for some $\bar{u} \in E_{J_{0}}$ and $T_{J_{0}}(-t) u \in E_{J_{0}}^{\varepsilon}$ then, to conclude that $T_{J_{0}}(-t) u \in U_{J_{0}}(\bar{u})$ when $\varepsilon$ is sufficiently small, it is enough to show that there exists $\delta>0$ such that $W_{J_{0}}^{u}(v) \cap B(v, \delta) \subset U_{J_{0}}(v)$, for all $v \in E_{J_{0}}$. But this result follows immediately from Lemma 18.

Theorem 23. Assume the hypotheses (H1), (H2), and (H5) with $a<\infty$ and (H6) and (H7) hold. Then the family of attractors $\mathscr{A}_{J}$ is lower semicontinuous with respect to the parameter $J$ at $J_{0} \in \mathcal{J}$.

Proof. Let $\varepsilon>0$ be given. From Lemma 22 there is a $T>0$ such that, for all $u \in \mathscr{A}_{J_{0}} \backslash E_{J_{0}}^{\varepsilon}$, there exists $t_{u} \in[0, T]$ such that

$$
\bar{u}:=T_{J_{0}}\left(-t_{u}\right) u \in U_{J_{0}}\left(u_{0}\right),
$$

for some $u_{0} \in E_{J_{0}}$. Since $T_{J_{0}}(t)$ is a continuous family of bounded operators there exists $\eta>0$ such that, for all $t \in$ $[0, T]$,

$$
\|z-w\|_{L^{2}}<\eta \Longrightarrow\left\|T_{J_{0}}(t) z-T_{J_{0}}(t) w\right\|_{L^{2}}<\frac{\varepsilon}{2} .
$$

Now, by the uniform continuity of the equilibria and the local unstable manifolds with respect to the parameter $J$ guaranteed by Theorem 16 and Lemma 19, there exists $\delta^{*}>$ 0 independent of $u$ such that $\left\|J-J_{0}\right\|_{L^{1}}<\delta^{*}$ implies the existence of $u_{J} \in E_{J}$ and some $\overline{\bar{u}}_{J} \in U_{J}\left(u_{J}\right)$ with

$$
\left\|\overline{\bar{u}}_{J}-\bar{u}\right\|_{L^{2}}<\eta
$$

where $U_{J}\left(u_{J}\right)$ denotes the local unstable manifold of the equilibrium $u_{J}$ of $T_{J}(t)$. Hence, when $\left\|J-J_{0}\right\|_{L^{1}}<\delta^{*}$ we obtain, from (110) and (111),

$$
\left\|T_{J_{0}}(t) \overline{\bar{u}}_{J}-T_{J_{0}}(t) \bar{u}\right\|_{L^{2}}<\frac{\varepsilon}{2}, \quad \text { for any } t \in[0, T] \text {. }
$$

On the other hand, from the continuity of the flow with respect to parameter $J$, there exists $\bar{\delta}>0$ such that $\left\|J-J_{0}\right\|_{L^{1}}<\bar{\delta}$ implies

$$
\left\|T_{J}(t)(u)-T_{J_{0}}(t)(u)\right\|_{L^{2}}<\frac{\varepsilon}{2},
$$

for any $u \in B(0,2 a \sqrt{2 \tau})$ and $t \in[0, T]$, and in particular for $u=\overline{\bar{u}}_{J}$ and $t=t_{u}$.

Consider $\delta=\min \left\{\delta^{*}, \bar{\delta}\right\}$ and let $v_{J}:=T_{J}\left(t_{u}\right) \overline{\bar{u}}_{J}$. It is clear that $v_{J} \in \mathscr{A}_{J}$ since $\overline{\bar{u}}_{J} \in U_{J}\left(u_{J}\right)$.

Using (112) and (113) we obtain

$$
\begin{aligned}
\left\|v_{J}-u\right\|_{L^{2}}= & \left\|T_{J}\left(t_{u}\right) \overline{\bar{u}}_{J}-T_{J_{0}}\left(t_{u}\right) \bar{u}\right\|_{L^{2}} \\
\leq & \left\|T_{J}\left(t_{u}\right) \overline{\bar{u}}_{J}-T_{J_{0}}\left(t_{u}\right) \overline{\bar{u}}_{J}\right\|_{L^{2}} \\
& +\left\|T_{J_{0}}\left(t_{u}\right) \overline{\bar{u}}_{J}-T_{J_{0}}\left(t_{u}\right) \bar{u}\right\|_{L^{2}} \\
<\varepsilon &
\end{aligned}
$$

provided that $\left\|J-J_{0}\right\|_{L^{1}}<\delta$.

When $u \in E_{J_{0}}^{\varepsilon} \subset \mathscr{A}_{J_{0}}$ this conclusion follows straightforwardly from the continuity of equilibria. Thus the lower semicontinuity of attractors follows.

\section{A Concrete Example}

In this section we illustrate the results of the previous sections to the particular case of (1) where $g(x)=\tanh (x)$.

In this case, we can rewrite $(\mathbf{P})_{J}$ as follows:

$$
\frac{\partial v(w, t)}{\partial t}=-v(w, t)+\tanh (\beta(J * v)(w, t)+\beta h)
$$

In this case, if $\beta \leq 1$ (115) has only one (stable) equilibrium (see [9]). If $\beta>1$, there is $h^{*}$, implicity defined by (116), such that, for $0 \leq h \leq h^{*}$, (115) has three equilibria, $m_{\beta}^{-}, m_{\beta}^{0}$, and $m_{\beta}^{+}$, each of which is identically equal to one of the roots of the equations:

$$
m_{\beta}=\tanh \left(\beta m_{\beta}+\beta h\right) .
$$

The Lyapunov functional for (115) is given by

$$
\begin{aligned}
\mathbb{F}(u)= & \int_{S^{1}}[f(u(w))-f(\bar{m})] d w \\
& +\frac{1}{4} \iint_{S^{1}} J\left(w \cdot z^{-1}\right)[u(w)-u(z)]^{2} d w d z,
\end{aligned}
$$


where $f$ (the free energy density) is given by

$$
f(x)=-\frac{1}{x^{2}}-h x-\beta^{-1} i(x), \quad x \in[-1,1],
$$

and $i$ is the entropy density, given by

$$
\begin{array}{r}
i(x)=-\frac{1+x}{2} \ln \left(\frac{1+x}{2}\right)-\frac{1-x}{2} \ln \left(\frac{1-x}{2}\right), \\
x \in[-1,1] .
\end{array}
$$

As was observed in $[4,8,9]$, the functional given in (117) has minimum value at $m_{\beta}^{+}$.

Note that $g$ satisfies (H1) and (H4) with $k_{1}=a=1$. Moreover, it is easy to see that (4) is satisfied with $k_{2}=1 / 3$ and $k_{3}=3$.

Now, we observe that $g \in C^{2}(\mathbb{R}), g^{\prime}(x)=\operatorname{sech}^{2}(x)$, and $g^{\prime \prime}(x)=-2 \operatorname{sech}^{2}(x) \tanh (x)$. Thus (H2), (H3), and (H7) hold. In particular

$$
\left|g^{\prime}(x)\right| \leq k_{4}|x|+k_{5}
$$

where $k_{4}=2$ and $k_{5}=0$ and $g^{\prime}(x) \leq b$ with $b=1$.

Therefore all results of the previous sections are valid for the particular case of the flow generated by (115).

\section{Conflict of Interests}

The authors declare that there is no conflict of interests regarding the publication of this paper.

\section{Acknowledgments}

The authors thank the anonymous referee for his/her careful reading of the paper and valuable suggestions. The first author is supported by CAPES/CNPq-Brazil, the second author is supported by INCTMat, and the third author is supported by FAPESP.

\section{References}

[1] S. R. M. Barros, A. L. Pereira, C. Possani, and A. Simonis, "Spatially periodic equilibria for a non local evolution equation," Discrete and Continuous Dynamical Systems. Series A, vol. 9, no. 4, pp. 937-948, 2003.

[2] A. Masi, E. Orlandi, E. Presutti, and L. Triolo, "Glauber evolution with the Kac potentials. I. Mesoscopic and macroscopic limits, interface dynamics," Nonlinearity, vol. 7, no. 3, pp. 633696, 1994.

[3] A. Masi, T. Gobron, and E. Presutti, "Travelling fronts in nonlocal evolution equations," Archive for Rational Mechanics and Analysis, vol. 132, no. 2, pp. 143-205, 1995.

[4] A. Masi, E. Orlandi, E. Presutti, and L. Triolo, "Uniqueness and global stability of the instanton in nonlocal evolution equations," Rendiconti di Matematica e delle sue Applicazioni. Serie VII, vol. 14, no. 4, pp. 693-723, 1994.
[5] A. Masi, E. Olivieri, and E. Presutti, "Critical droplet for a nonlocal mean field equation," Markov Processes and Related Fields, vol. 6, no. 4, pp. 439-471, 2000.

[6] A. Masi, E. Orlandi, E. Presutti, and L. Triolo, "Stability of the interface in a model of phase separation," Proceedings of the Royal Society of Edinburgh: Section A Mathematics, vol. 124, no. 5, pp. 1013-1022, 1994.

[7] A. L. Pereira and S. H. da Silva, "Existence of global attractors and gradient property for a class of non local evolution equations," São Paulo Journal of Mathematical Sciences, vol. 2, no. 1, pp. 1-20, 2008.

[8] A. L. Pereira and S. H. da Silva, "Continuity of global attractors for a class of non local evolution equations," Discrete and Continuous Dynamical Systems. Series A, vol. 26, no. 3, pp. 10731100, 2010.

[9] A. L. Pereira, "Global attractor and nonhomogeneous equilibria for a nonlocal evolution equation in an unbounded domain," Journal of Differential Equations, vol. 226, no. 1, pp. 352-372, 2006.

[10] H. Brezis, Análisis Funcional Teoría y Aplicaciones, Alianza, Madrid, Spain, 1984.

[11] J. L. Daleckii and M. G. Krein, Stability of Solutions of Differential Equations in Banach Space, vol. 43, American Mathematical Society, Providence, RI, USA, 1974.

[12] J. K. Hale, Asymptotic Behavior of Dissipative Systems, vol. 25 of Mathematical Surveys and Monographs, American Mathematical Society, Providence, RI, USA, 1988.

[13] J. M. Arrieta and A. N. Carvalho, "Spectral convergence and nonlinear dynamics of reaction-diffusion equations under perturbations of the domain," Journal of Differential Equations, vol. 199, no. 1, pp. 143-178, 2004.

[14] A. N. Carvalho and S. Piskarev, "A general approximation scheme for attractors of abstract parabolic problems," Numerical Functional Analysis and Optimization, vol. 27, no. 7-8, pp. 785-829, 2006.

[15] J. K. Hale and G. Raugel, "Lower semicontinuity of attractors of gradient systems and applications," Annali di Matematica Pura ed Applicata, vol. 154, no. 1, pp. 281-326, 1989.

[16] L. A. F. Oliveira, A. L. Pereira, and M. C. Pereira, "Continuity of attractors for a reaction-diffusion problem with respect to variations of the domain," Electronic Journal of Differential Equations, no. 100, pp. 1-18, 2005.

[17] A. L. Pereira and M. C. Pereira, "Continuity of attractors for a reaction-diffusion problem with nonlinear boundary conditions with respect to variations of the domain," Journal of Differential Equations, vol. 239, no. 2, pp. 343-370, 2007.

[18] P. W. Bates, K. Lu, and C. Zeng, Existence and Persistence of Invariant Manifolds for Semiflows in Banach Space, vol. 645 of Memoirs of the American Mathematical Society, American Mathematical Society, Providence, RI, USA, 1998.

[19] S. H. da Silva and A. L. Pereira, "Exponential trichotomies and continuity of invariant manifolds," São Paulo Journal of Mathematical Sciences, vol. 5, no. 2, pp. 111-124, 2011.

[20] J. K. Hale and G. Raugel, "Convergence in gradient-like systems with applications to PDE," Zeitschrift für Angewandte Mathematik und Physik, vol. 43, no. 1, pp. 63-124, 1992.

[21] S. H. da Silva, "Lower semicontinuity of global attractors for a class of evolution equations type neural fields in a bounded domain," http://xxx.tau.ac.il/abs/1312.6745. 
[22] D. Henry, Evolution Equations in Banach Spaces, Notes in Mathematics-IME, Universidade de São Paulo, São Paulo, Brazil, 1981.

[23] E. N. Dancer, "The G-invariant implicit function theorem in infinite dimensions," Proceedings of the Royal Society of Edinburgh: Section A Mathematics, vol. 92, no. 1-2, pp. 13-30, 1982. 


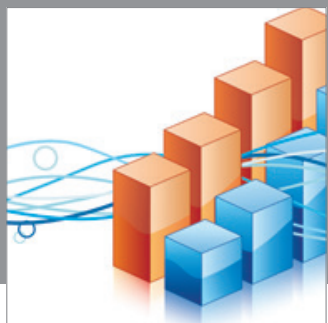

Advances in

Operations Research

mansans

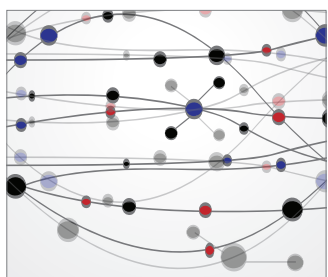

The Scientific World Journal
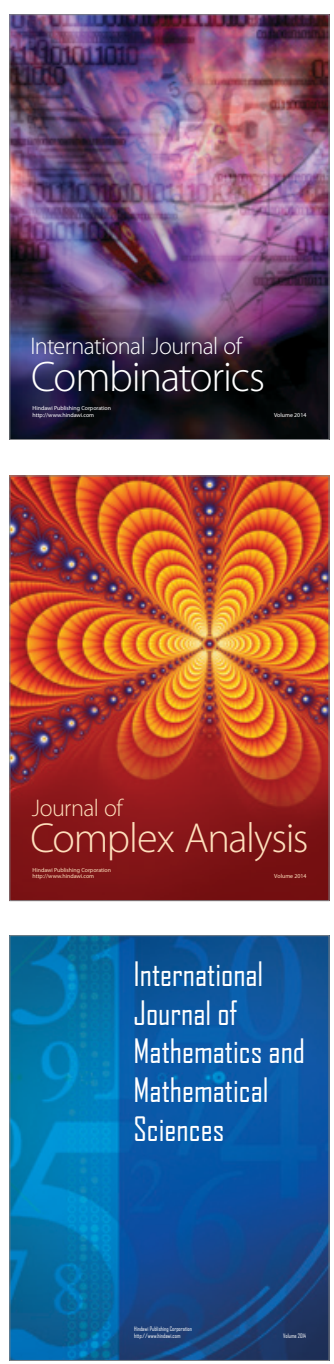
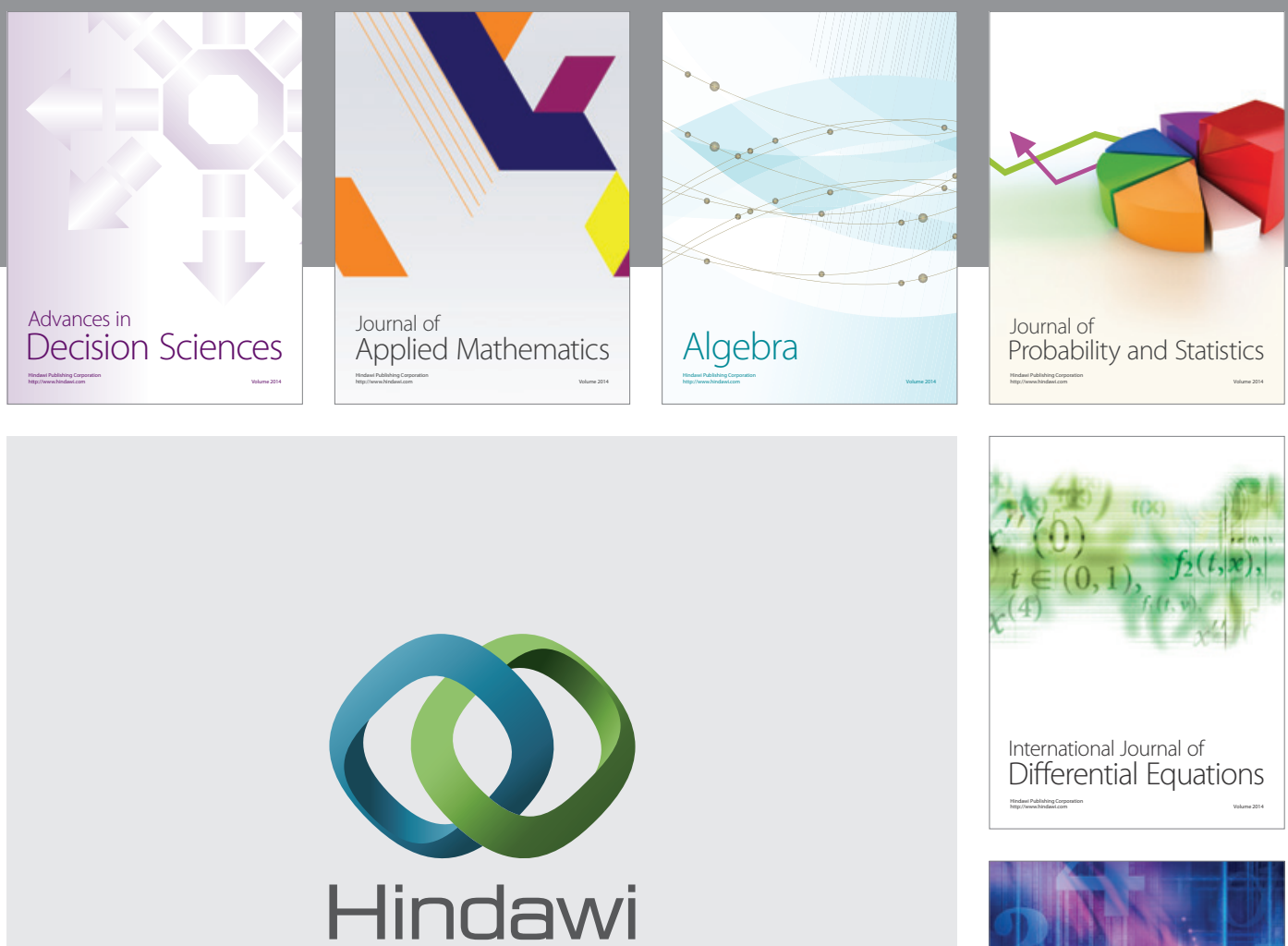

Submit your manuscripts at http://www.hindawi.com
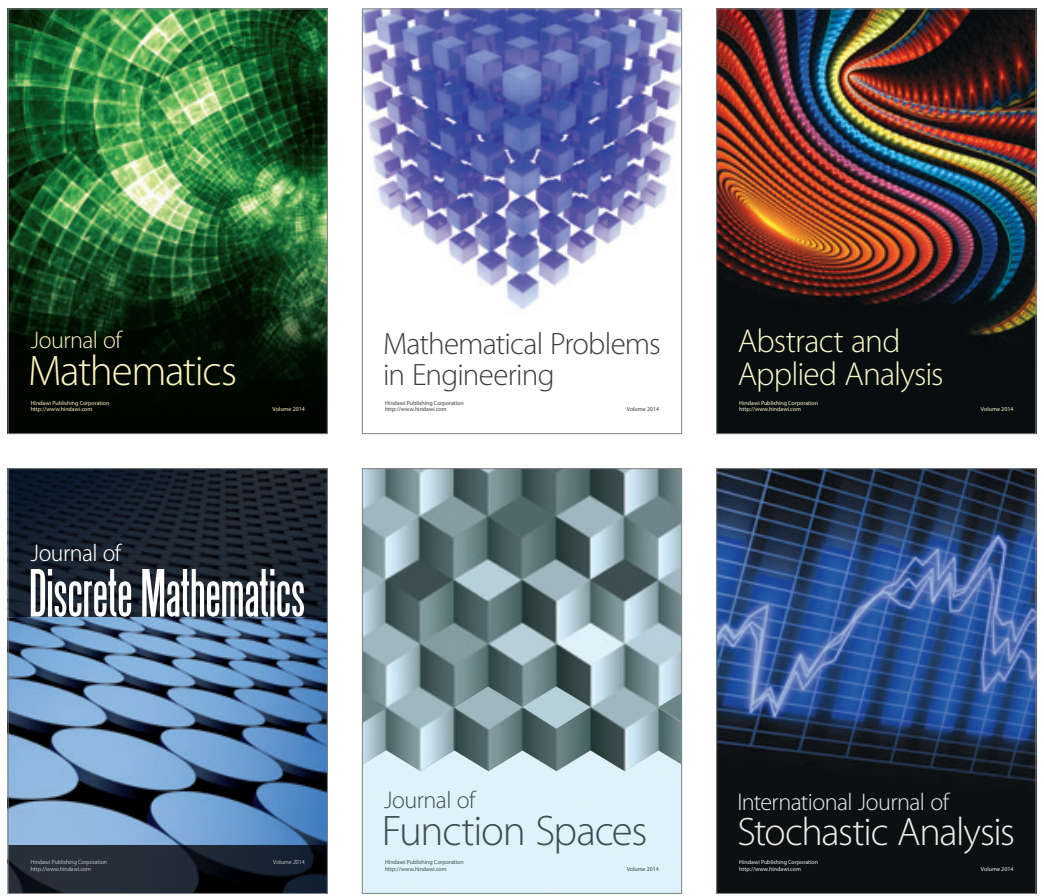

Journal of

Function Spaces

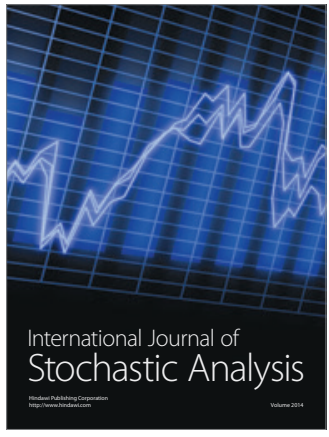

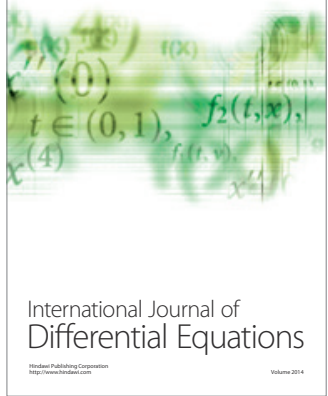
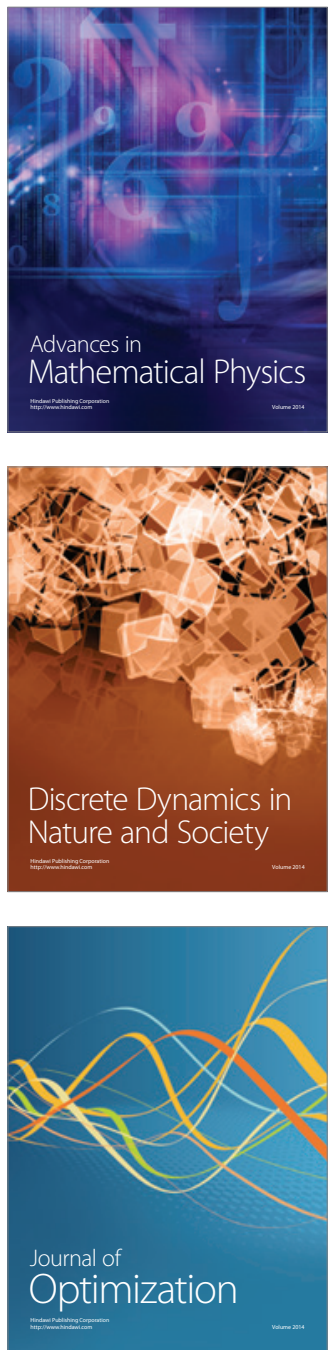